\title{
Massimo Bontempelli giornalista e ammiratore dei Quattrocentisti: per un rinnovamento letterario della Terza epoca
}

\author{
Massimo Bontempelli, Journalist and Admirer of the Italian \\ Quattrocento: Towards a Literary Renewal for the Third Era
}

Dana Božič

Università di Lubiana, Slovenia

\begin{abstract}
Riassunto: L'articolo presenta alcuni testi di Massimo Bontempelli finora relativamente poco esplorati dalla critica bontempelliana o perfino sconosciuti, provenienti dall'ambito del giornalismo e da collaborazioni editoriali. L'analisi della prefazione alle Prose di fede e di vita del primo tempo dell'umanesimo, scritta da Bontempelli nel 1913, mostra (anche al confronto con altri articoli bontempelliani sia del periodo degli esordi, sia dei primi anni Venti) che numerose tesi della tendenza culturale e letteraria del 'novecentismo' teorizzata tra il 1926 e il 1927, erano presenti già nelle prime collaborazioni editoriali di questo intellettuale. Fra queste tesi: il ruolo di una lingua trasparente, l'importanza dell'immaginazione, la necessità di prestare attenzione al pubblico e di liberarsi da una troppo stretta adesione al canone, per nominarne alcune.

Parole chiave: Massimo Bontempelli, novecentismo, Quattrocento letterario, prefazione, giornalismo.
\end{abstract}

Abstract: The article presents selected newspaper articles and a preface written by Massimo Bontempelli which have been relatively unexplored and unacknowledged by the literary criticism. The comparative analysis of the preface from 1913 and selected articles from his early period and the early 1920s, shows that numerous core ideas pertaining to his cultural and literary project 'novecentismo', which was officially launched as a theoretical proposal between 1926-1927, were already present in his early editorial collaborations as well as the articles: the role of linguistic transparency, the importance of imagination, Bontempelli's anti-canonical attitude and the role he assigns to the reader, to name a few.

Keywords: Massimo Bontempelli, novecentism, the Quattrocento, preface, journalism.

\section{Introduzione}

Massimo Bontempelli (1878-1960) scrittore, drammaturgo, poeta, compositore, giornalista e traduttore italiano, è noto per il suo impegno di rinnovamento culturale e 
letterario con il quale intendeva svecchiare lo spazio culturale e letterario italiano dopo il primo conflitto mondiale mettendosi nel vortice del dibattito culturale del Paese. Gli studi dedicatigli hanno messo in luce aspetti della sua opera narrativa, poetica, teatrale ed evidenziato il suo ruolo di teorico e diffusore del realismo magico in Italia; minore attenzione è stata invece finora assegnata al suo lavoro di collaboratore editoriale e pubblicista. Ad esempio il suo lavoro sulle prefazioni, nell'ambito della produzione critico-letteraria, rappresenta un terreno collaterale di attività, che risulta altrettanto pertinente indagare perché rivela, tra l'altro, che numerose posizioni bontempelliane sulla cultura, arte e letteratura erano maturate oltre un decennio prima della teorizzazione della tendenza novecentista.

\title{
1. L'oggetto e le ipotesi del lavoro
}

Nel presente articolo, attraverso l'analisi di un lavoro critico di Bontempelli risalente al 1913 relativo a una collaborazione editoriale, nonché di alcuni altri suoi articoli apparsi su alcuni quotidiani tra il 1908 e gli anni Venti, intendiamo dimostrare che certi tratti della poetica novecentista erano presenti già molti anni prima del lancio ufficiale nel 1926, e non solo a partire dagli anni Venti come è stato in gran parte indicato dalla critica letteraria. Si tratta, nel caso del lavoro del 1913, di una Introduzione che Bontempelli scrisse alle Prose di fede e di vita del primo tempo dell'umanesimo raccolta di testi in prosa ad uso scolastico, di cui Bontempelli era curatore. Con quel testo prefatorio egli presentava un panorama su testi in volgare di generi in prosa praticati nella penisola italiana nel primo Quattrocento: cronache, lettere, saggi, prediche. Si tratta di un periodo in cui, nel contesto italiano, la lingua letteraria principale era tornata ad essere il latino, mentre il volgare era relegato ad usi pratici e a opere di destinazione popolare, non essendo quindi considerato la lingua della cultura. Con la scelta dei testi non canonici e soprattutto connessi a funzioni private, Bontempelli avrebbe presumibilmente cercato non solo di promuovere le proprie idee, ma anche di avvicinarsi al pubblico più giovane, il quale, se avesse seguito fedelmente il canone proposto dalle autorità scolastiche, avrebbe potuto trovare lo studio della letteratura faticoso e noioso. Un tale pubblico per Bontempelli avrebbe rappresentato un terreno di ricezione ancora incontaminato dalle interpretazioni della critica avvalorata e dalle definizioni letterarie proposte nell'ambito scolastico, terreno costituito da persone capaci quindi di leggere e interpretare sia i classici che i minori in un modo genuino e senza pregiudizi. Su questo primitivismo delle menti giovani e sulla importanza della lettura dei classici si esprimerà nel 1927, in uno degli articoli programmatici del novecentismo, intitolato Consigli:

\begin{abstract}
Occorre leggere da giovani i grandi capolavori del passato, per non doverli leggere più tardi, quando c'è altro da fare. E soprattutto per questo: che nella maturità ce li troveremo diventati nostro patrimonio come ripensati, rievocati, sgombri delle parole. In questo modo la Commedia o il Furioso sono grandissimi nell'animo nostro (Bontempelli, 1938, p. 30).
\end{abstract}

L'oggetto principale del nostro lavoro sarà tracciare le idee novecentiste presenti nella prefazione del 1913 e in alcuni articoli pubblicati soprattutto prima della teorizzazione ufficiale del novecentismo: intendiamo mostrare che sia la prefazione in questione, che gli articoli che affronteremo qui, trattano temi simili a quelli di cui Bontempelli parlerà nella teorizzazione del suo progetto del rinnovamento culturale e letterario, il novecentismo, divulgato sulla rivista «900» a partire dall'autunno del 1926; aspetti che egli andava maturando già da tempo, come provano pure altri suoi scritti 
fra cui la prefazione a II Poliziano, II Magnifico. Lirici del Quattrocento' del 1910. Questo almeno quanto è emerso finora nella ricerca che stiamo sviluppando nell'ambito di uno studio di tesi dottorale. L'ipotesi di fondo che guida anche il presente contributo è, appunto, che le posizioni novecentiste maturino ben prima della loro teorizzazione e che questo avvenga anche attraverso scritti di critica letteraria, come sono recensioni e prefazioni. Quali posizioni, nello specifico? Parliamo dell'importanza del dilettantismo dal quale può nascere un capolavoro, dell'ispirazione intuitiva, dell'atteggiamento antiaccademico contro il canone, della tesi di un rinnovamento culturale nel ritorno al primordiale, della difesa della portata sovratemporale delle opere classiche e della necessità di una letteratura capace di uscire dal proprio spazio e tempo, nonché della tesi del basilare ruolo del lettore.

Rileveremo che, similmente a quanto già emerso da una analisi dell'Introduzione ai Lirici, Bontempelli assume anche nei testi che affronteremo qui una retorica spigliata e viene sviluppando un discorso approfondito sul Quattrocento proprio per esprimere la sua predilezione per la letteratura di quella fase, allo stesso tempo, però, adegua il suo stile al pubblico di referenza e non assume quei termini irriverenti che a tratti appaiono invece nei suoi articoli critici e letterari pubblicati su periodici e quotidiani come ad esempio sulla rivista Rassegna contemporanea nel 1908 e sul Marzocco del $1910^{2}$.

L'intervento affronta una lettura di carattere socio-culturale, rilevando in particolare quale rapporto il novecentismo, già nella sua fase di preparazione, istituisca fra i produttori di arte e letteratura, da un lato, e i loro ricettori dall'altro, e inoltre quale tipo di cultura esso sostenga. II discorso affrontato in questo contesto si sviluppa poi attraverso confronti intratestuali concernenti l'opera critica bontempelliana, tra gli interventi (ancora per nulla studiati) del periodo 1913-1922, pubblicati appunto in una fase di elaborazione di tesi che andranno poi a delineare la tendenza novecentista e quelli, più noti, risalenti agli anni 1926-1927, in cui ebbe luogo la teorizzazione e il lancio di tale proposta di rinnovamento culturale.

\section{Le dinamiche editoriali intorno al 1913}

Il soggiorno fiorentino (1910-1914) di Bontempelli segna la rinuncia (dopo il fallimento in due concorsi per l'accesso all'insegnamento dell'italiano nei licei) a una carriera nella scuola e la decisione di dedicarsi alla scrittura e alle traduzioni a tempo pieno. Si tratta senz' altro di un passo rischioso che non promette una fonte di reddito sicura. Come nota Tranfaglia, Firenze in quel periodo diventa "capitale delle riviste e dei

\footnotetext{
1 Questa collaborazione editoriale sarebbe stata una delle iniziative di quel genere. Cfr. Bontempelli, $1910 \mathrm{~b}$

2 Si veda: Bontempelli, 1908b. Nel caso del quotidiano II Marzocco, si tratta di tre articoli che sono pure oggetto della nostra: Bontempelli, M. (1910). Un nuovo e un antico. Il Marzocco, 8 maggio 1910, n. 19, a. XV, pp. 2-3.; Bontempelli, M. (1910). Fuori della letteratura. Il Marzocco, 29 maggio 1910, n. 22, a. XV, p. 4.; Bontempelli, M. (1910). In difesa dei pedanti: Per un libro di F. D'Ovidio. II Marzocco, 26 giugno 1910 , n. 26, a. XV, pp. 3-4.

In quegli articoli, l'autore quando si riferisce alla produzione contemporanea e ai suoi protagonisti adotta a volte campi semantici pertinenti al corpo umano e alla salute. Scrive ad esempio recere in "Grande e piccola critica", oppure malato in "Un nuovo e un antico", mentre mantiene un tono piuttosto rispettoso (anche se critico) riferendosi ad autorità letterarie, come Croce e De Sanctis. Quindi, benché Bontempelli esprima spesso posizioni antiaccademiche, non sempre adotta un linguaggio irriverente. Lo stesso vale per l'ironia: essa traspare di frequente dai suoi articoli, mentre non accade altrettanto nel testo prefatorio ai Lirici che rivela un Bontempelli rispettoso della letteratura classica.
} 
tentativi di molti intellettuali" (citato in Tranfaglia \& Vittoria, 2000, p. 22) ed è un punto di partenza anche per Bontempelli. La scelta attuata dalla casa editrice Sansoni di affidargli un ruolo editoriale comporta dei vantaggi per entrambe le parti coinvolte. ${ }^{3}$ In questo periodo si nota in Italia una nuova tendenza editoriale, dovuta alla riforma scolastica degli ultimi decenni dell'Ottocento, il cui obiettivo era stato quello di realizzare una formazione dei giovani che prevedesse il rapporto diretto con il testo, e i cui risvolti coinvolgono all'inizio del secolo numerosi intellettuali, scrittori, giornalisti e poeti italiani. L'incarico editoriale affidato a Bontempelli dunque non è un caso isolato. Secondo Giuseppe Ragone quella nuova tendenza pretende allora che si studino i testi letterari in forma integrale senza commenti e spiegazioni, e con lo scopo di una migliore formazione culturale e linguistica, ovvero di una "formazione letteraria" (citato in Asor Rosa, 1983, p. 710) meno finalizzata agli stretti obiettivi scolastici. Una tale scelta è giustificata con la tesi che il testo letterario è all'epoca sempre più contaminato dal settore giornalistico e "dalla nuova geografia culturale del "consumo»" (Asor Rosa, 1983, p. 710).

\section{La prefazione alle Prose, il Diorama e la ricerca della fantasia}

La prefazione sin da subito rivela le posizioni anticanoniche di Bontempelli, le quali si svelano qui attraverso delle osservazioni pertinenti sia al genere della poesia, sia a quello della prosa. II suo ragionamento emerge da una semplice constatazione su come la poesia e la prosa vengono generalmente percepite: "La poesia è fantasia, la prosa è ragionamento [...]" (Bontempelli, 1913, p. III). Osserva che la distinzione fra le due nella storia della letteratura è stata spesso fraintesa ovvero pensa che essa "abbia influito sinistramente sulla prosa, intesa in altro senso, più materiale e ristretto" (Bontempelli, 1913, p. III). Per questo motivo i due generi sono sempre stati ritenuti imparagonabili, ma soprattutto la prosa ha sempre goduto di meno prestigio della lirica. Bontempelli afferma: "Anche l'oratio soluta è poesia [...]" mettendola così nella stessa categoria della lirica. Affinché questo succeda ci sono però alcune condizioni: "I'immediatezza, la perspicuità, la vivacità creativa d'immagini, la semplicità dei mezzi" (Bontempelli, 1913, p. III).

La questione circa le differenze e le analogie tra i generi della lirica e della prosa si lega ad alcune osservazioni che egli svilupperà in un articolo intitolato Che cosa è «poesia» del 1931 sul Diorama della Gazzetta del Popolo (Bontempelli, 1938, pp. 96103). Rendendola quasi una querelle, ne parlerà in un articolo specifico e lo farà rivolgendosi in modo irriverente al supplemento del quotidiano, personificandolo: "Caro Diorama, tu vuoi farmi morire di rabbia. Vuoi trascinarmi per i capelli nella più traditoria delle discussioni. E tu vuoi far ridiventare crociani per forza tutti coloro che Croce se l'erano ben digerito ed erano andati avanti" (Bontempelli, 1938, p. 96). In un'inchiesta apparsa su Diorama, la redazione chiedeva ai lettori, attraverso un questionario, di dare una definizione di 'poesia' e (ipotizziamo) anche di 'prosa'. Bontempelli, toccato dalla questione - perché essa stava al cuore delle sue proposte di rinnovamento, ma anche perché amareggiato che l'inchiesta partisse da una pubblicazione che sarebbe dovuta essere essa stessa la prima a promuovere le soluzioni per il futuro letterario e culturale - risponde indignato al questionario. Lo sfogo

\footnotetext{
${ }^{3}$ La casa editrice Sansoni, specializzata nelle pubblicazioni scolastiche ed universitarie, guadagna in tal modo un esperto sul campo della filologia e allo stesso tempo anche un ex insegnante consapevole delle esigenze dei giovani lettori e delle dinamiche dell'insegnamento; dall'altra parte, Bontempelli riesce ad ottenere, a parte una fonte di reddito, anche una piattaforma ulteriore per sviluppare le sue idee, ad inserirsi nell'ambito editoriale e letterario, nonché per espandere la sua rete di conoscenze nel settore.
} 
polemico ma allo stesso tempo costruttivo di Bontempelli come risposta alla redazione di Diorama riguarda appunto la percezione dell'apparente differenza tra la prosa e la poesia, che neanche i lettori sono capaci di distinguere. La risposta di Bontempelli ricorda vivamente le osservazioni sulla critica e sulla produzione letteraria che egli aveva sviluppato nell'articolo Grande e piccola critica del 1908, ma anche sugli articoli apparsi sul Marzocco nel 1910, ad esempio in In difesa dei pedanti: per un libro di F. D'Ovidio. Avverso alle definizioni e al pedantismo oltre che all'influsso che un testo come la Poetica di Aristotele poteva avere sull'opera estetica, una "congerie di osservazioni ingabbiate in categorie, [...] [orientata alla] costruzione esatta e immobile di norme per un numero determinato di generi letterari" (Bontempelli, 1908b, p. 131), come descriveva la Poetica di Aristotele nel 1908, Bontempelli persino ancora nel 1931 sosterrà che proprio un tipo di critica normativa e basata su rigorose distinzioni ha fortemente influenzato i lettori, che hanno "sempre avuto la tendenza a restringere il campo della parola: poesia." E continua: "C'è ancora oggi della gente la quale crede che "poesia» voglia dire scrivere in versi; poesia la Commedia, non il Decamerone [...]" (Bontempelli, 1938, p. 96). Di conseguenza Bontempelli lancia una domanda a Diorama, ovvero ai suoi redattori: "La cosiddetta lirica e la cosiddetta arte narrativa, non adempiono forse al medesimo compito?" (Bontempelli, 1938, p. 98). Entrambe potrebbero essere chiamate «poesia», che per Bontempelli significa "uarte dello scrivere», in contrapposizione alle altre arti (arti del disegno, musica)" (Bontempelli, 1938, p. 97).

Nel 1931 il teorico del novecentismo si indignava dunque che una tale concezione della prosa e della lirica fosse rilanciata da Diorama, una pubblicazione tipica dei mass media del tempo con la funzione di diffondere la cultura. Della questione se ne era occupato tuttavia già nell'Introduzione alle Prose del 1913 dove era andato a scavare più profondamente nella storia della letteratura italiana. La colpa di una tale durevole demarcazione tra la lirica e la prosa viene attribuita, in quel contesto, a Giovanni Boccaccio, accusato di aver assunto lo stile ciceroniano come modello per la sua prosa, il che avrebbe avuto delle gravi conseguenze su tutta la letteratura italiana successiva e dalla quale ancora nel presente essa cercava di liberarsi (Bontempelli, 1913, p. III). Questo avvelenamento non ha colpito solo la trattatistica trecentesca, ma perfino la narrativa. I difensori del canone, ovvero i "trattatisti, ci danno [perciò] come periodo aureo della prosa l'insopportabile [C] inquecento, e affermano che nella prima metà del quattrocento troviamo un salto, una lacuna: che per mezzo secolo almeno non si scrisse più prosa d'arte" (Bontempelli, 1913, pp. III-IV)4.

Quindi, in un certo senso, nell'Introduzione alle Prose, Bontempelli è critico della demarcazione che si era fatta tra la lirica e tra la prosa, e di conseguenza dello status inferiore che la prosa aveva guadagnato rispetto alla lirica, perché più "comune" (Bontempelli, 1913, p. III). Per questo aspetto, Bontempelli è molto vicino a Benedetto Croce che, nella sua Estetica, nel 1902 condannava la divisione in generi letterari: "il maggior trionfo dell'errore intellettualistico è nella teorica dei generi artistici letterari, che ancora corre nei trattati e perturba i critici e gli storici dell'arte" (Croce, 1908, p.

\footnotetext{
${ }^{4}$ A questo punto va notato che Bontempelli negli anni successivi scriverà delle prefazioni a opere di autori provenienti proprio da quell'ambito, ad esempio a Giovan Battista Gelli, un "calzolaio erudito", appassionato dei classici, e i cui scritti saranno lodati per il loro "contatto continuo con la vita, con l'umile realtà quotidiana [...]" e per il "suo senso schietto e volenteroso del popolo, che sa accomodarsi una via di mezzo in tutto: tra l'incultura e l'erudizione, tra la pratica e la scienza, tra l'eresia [...]" (Bontempelli, 1916, p. 21)
} 
42). Per Croce, qualsiasi forma letteraria non ha bisogno dell'espressione perché "è già essa stessa espressione" (Croce, 1908, p. 43). Inoltre, sia per Bontempelli che per Croce era importante anche la formazione dello scrittore (o di un artista), il "padroneggiamento della tecnica" (Croce, 1905), come lo chiamava, e delle esperienze che egli fa nel corso della sua vita ${ }^{5}$. Per entrambi però, l'opera letteraria perde il suo valore estetico appena le impressioni vengono sottoposte alla "elaborazione delle espressioni" (Croce, 1908, p. 58). Da ciò traspare la loro predilezione per una letteratura che nasce dall'intuizione, spontaneamente, senza le costrizioni del genere o del canone.

Quindi nel primo paragrafo della prefazione alle Prose, che come tipo di testo ha la funzione di parlare direttamente al lettore (Genette, 1989, p. 3), Bontempelli dimostra l'intento di innalzare il genere della prosa e di mostrare che essa merita lo stesso valore e ammirazione che ha la lirica per le sue capacità trasfigurative.

Prima di indagare su come Bontempelli eseguirà un lavoro che dia giustizia alla grandezza della prosa dei quattrocentisti trascurati dal canone letterario, va aperta una piccola parentesi sul motivo per cui egli spesso, nelle collaborazioni editoriali, scelga e presenti autori minorib, meno conosciuti o perfino sconosciuti, o riconosciuti solo dopo la loro morte (Baretti, Stendhal 7 ). L'avversione per il canone letterario non l'avrebbe potuta spiegare meglio nessun altro che lo stesso Bontempelli, cosa che fece in un articolo intitolato "Cronache della prosa - Cercando la fantasia" che appare nel 1922 nel periodico $/ /$ Mondo. $^{8}$

In quell'articolo egli si avvia alla ricerca della fantasia negli autori contemporanei e recensisce alcune delle loro opere (romanzi, raccolte di racconti). Bontempelli concepisce l'arte moderna come un'arte che "respiri e divaghi e si abbandoni mobilmente spaziando tra [...] due compiti [...]" (Bontempelli, 1922): I'attivazione della fantasia e la consolazione. Questo dinamismo è caratterizzato soprattutto dal problema se l'arte possa raggiungerlo attraverso un "libero esercizio della immaginazione e il riaccostamento alle fonti più semplici ed elementari della vita; e per l'una e per l'altra via riesca a sfuggire la quotidiana realtà" (Bontempelli, 1922). Tutto ciò ricorda le posizioni novecentiste che saranno realizzate in campo letterario in base al realismo magico solo alcuni anni più tardi: "Unico strumento del nostro lavoro sarà l'immaginazione" (Bontempelli, 1938, p. 22).

Come afferma Bontempelli nell' articolo dal titolo "Cercando la fantasia", del 1922, la fantasia si raggiunge attraverso numerosi strumenti che Bontempelli ritrova nell'opera di quattro scrittori agli esordi: Ornella Quercia Tanzanella, Carlo Valcarenghi, Lucio d'Aquara, e Alfredo Mazza. Ad esempio, lo sforzo letterario di Carlo Valcarenghi, un giovane scrittore di meno di venticinque anni, è apprezzabile perché "di giovinezza, cioè di sfogo", ma Bontempelli ironizza sul motivo per cui Valcarenghi avrebbe scritto il romanzo Abracadabra: spera che sia di "liberazione,

\footnotetext{
${ }^{5}$ Si veda il suo articolo Varietà (Croce, 1905).

${ }^{6}$ Nella nostra ricerca dottorale ci siamo occupati anche del testo curato da Bontempelli delle Commedie e Satire di Ariosto, intitolato L'Ariosto minore.

${ }^{7}$ Si tratta di due collaborazioni editoriali che abbiamo pure preso in analisi: il lavoro sul romanzo II rosso e il nero di Stendhal del 1913, e sulla La frusta letteraria di Giuseppe Baretti.

${ }^{8}$ L'articolo fa parte della rubrica Cronache di prosa che Bontempelli tenne per il quotidiano in quegli anni. Alcuni altri articoli che siamo riusciti a reperire presso il Fondo Falqui (Biblioteca Nazionale di Roma) e che appartengono alla serie sono: "Raccontatori in corto" (03/11/1922), "Il vento nella foresta" (28/11/1922), "Un romanzo e 45 novelle" (01/03/1923), "Il libro del "«Quivis)" (s.d.), "Finestre alte" di Ada Negri (s.d.), "Narratori contemporanei" (s.d.), e "I ricordi di Ferdinando Martini" (s.d.).
} 
dai peggiori dei vizi di cui s'inquinò l'educazione della generazione ultima", e cioè del "decadentismo" perché (l'unico) esito del romanzo è "uno scetticismo [...] così baldanzoso [...], tanto è l'ingegno che circola anche nelle più inutili tra queste pagine" (Bontempelli, 1922). Non è però solo l'insistere sulle vecchie correnti letterarie di cui è critico Bontempelli: gli scrittori vengono, spesso attraverso un tono ironico, accusati della mancanza del dinamismo, alcuni dell'eccessiva digressione e della discussione a ogni costo, e dell'intreccio troppo facile. L'errore di non aver dato abbastanza spazio all'azione nel testo narrativo ricorda, ad esempio, la recensione alla Canzone dell'olifante pascoliana del 1908, scritta da Bontempelli nel 1908', ma soprattutto il novecentismo degli anni Venti: una delle forme predilette sarà appunto il romanzo d'avventure. ${ }^{10}$

Torniamo ora alla questione che ci interessa - alle scelte bontempelliane riguardo gli autori minori, sconosciuti e trascurati dal canone. I motivi per una tale valutazione da parte di Bontempelli di giovani scrittori agli esordi sono due. Scriverne un giudizio gli permetteva di giustificare le sue scelte curatoriali ed editoriali, ma anche, di esprimere attraverso quattro esempi rilevanti la sua frustrazione per la produzione letteraria contemporanea che era a suo giudizio ancora legata alle tendenze del secolo scorso. Si riporta qui nel seguito l'inizio dell'articolo "Cercando la fantasia" (non ancora riscoperto dalla critica bontempelliana), nel quale si rilevano appunto quei due aspetti:

Rispetto e venero tutti i nostri maggiori e più noti romanzieri, dei viventi intendo; i quali tutti mi sembrano eccellenti e degni di ammirazione. Ma il mio più ansioso interesse si volge ai minori, voglio dire quelli che son tali perché ancora giovani e alle prime prove nell'arte loro: minori e più oscuri oggi, saranno essi i più noti e maggiori domani (Bontempelli, 1922) ${ }^{11}$.

Gli autori minori, i giovani, gli sconosciuti proprio perché ancora non entrati nel canone, hanno il potenziale di rappresentare lo spirito del proprio tempo, nonché di anticipare quello futuro. Bontempelli nell'articolo cerca di dimostrare, attraverso l'analisi di quattro autori a lui contemporanei, che essi, essendo agli esordi in un momento storico e storico-letterario particolare, e perché appunto alle soglie della nuova epoca, hanno la possibilità e anche il sacro dovere di essere un esempio dello spirito del tempo per offrire i nuovi miti ai posteri, quindi sono i portatori del rinnovamento. Qualche anno prima, nella prefazione a La vita intensa, del 1919, nel

\footnotetext{
9 Bontempelli nel 1908 ne recensisce la Canzone dell'olifante criticando l'opera per alcune carenze a livello di struttura, per monotonia e per l'uso di artifizi troppo complessi in cui il lettore si perde.

$10 \mathrm{Si}$ veda il primo degli articoli programmatici del novecentismo intitolato "Giustificazione" ne L'Avventura novecentista (Bontempelli, 1938, pp. 18-19).

${ }^{11}$ E l'articolo continua: "Tale mia condizione di spirito non deriva da un ambizioso desiderio di essere in avanzo sui tempi. Nasce dal fatto, che gli autori maggiori e più noti rappresentano non già lo spirito odierno, ma quello di ieri; tendono cioè fatalmente a perpetuare situazioni più esaurite. Ognuno di essi sono quattro o cinque, è inutile ripeterne i nomi - sta correndo la seconda parte della sua parabola: raggiunte da tempo le proprie espressioni personali già compiute, ora non fa altro che lavorarvi attorno di variazioni; dacché nessuno d'essi ha la virtù, o le materiali possibilità, o la provvidenziale pigrizia che soccorse a tempo Giovanni Verga dal frantumare e diluire in dilettevoli e anacronistiche esercitazioni gli stati d'animo e d'arte di cui aveva trovata al tempo giusto l'espressione totale e perfetta.

Così stando le cose - e nessuno ignora che stanno così - chi si trovi, per gusto o per volgere di casi, a doversi occupare della produzione contemporanea, è tratto naturalmente a volgere la sua intenzione ai comincianti. Può essere più fecondo trovare in un autore inesperto qualche indicazione sul domani, che divertirsi a esaminare e proclamare per la ventesima volta le virtù psicologiche, o narrative, 0 descrittive di autori espertissimi e illustri" (Bontempelli, 1922).
} 
tentativo di compiere proprio quel rinnovamento, Bontempelli, conscio della sua responsabilità e della necessità di rinnovamento riguardo alla letteratura italiana, affermava: "Lo scrivo per i posteri. Lo scrivo per rinnovare il romanzo europeo" (Bontempelli, 2009, p. 7). Ancora nei Preamboli, in uno di quattro articoli programmatici del novecentismo, intitolato "Analogie", nel 1927, sosterrà: "il capolavoro è quell'opera che, finito il tempo in cui essa nacque, si trova ad avere la forza di uscire dalla portata di esso tempo, e a poter servire i posteri: del che soltanto essi i posteri sono giudici, e noi non dobbiamo né possiamo occuparcene, che è tempo perso [...]" (Bontempelli, 1938, p. 40). Bontempelli non è un postero rispetto ai giovani sottoposti all'esame, quindi non può giudicare ancora se le loro opere riusciranno ad uscire dal proprio tempo, quello però che può fare è di indagare sulle componenti delle loro opere e constatare se posseggono quelle che, secondo le posizioni novecentiste, un potenziale capolavoro dovrebbe avere. La delusione per i narratori contemporanei, ancora intorno al 1922, è per lui totale: Bontempelli non riesce a trovare nei loro testi alcun elemento interessante e innovativo perché essi continuano a risultare epigoni, a prendere come modello autori canonici invece di usare la loro immaginazione per produrre qualcosa di originale.

Tornando quindi alla nostra prefazione alle Prose, vediamo che già nel 1913 I'intento di Bontempelli, di includere autori e opere minori e sconosciute fra le letture da proporre ai giovani era mosso dalle stesse premesse. Inoltre appare evidente come già nel periodo dei suoi esordi egli si rendesse conto dell'ostacolo che il canone letterario rappresentava non solo al rinnovamento letterario, ma anche e soprattutto al rinnovamento della concezione del tempo e dello spazio, uno dei compiti più importanti che si sarebbe posto il novecentismo (Bontempelli, 1938, p. 17). Cercando dunque di ricostruire lo spirito quattrocentesco per presentarlo agli studenti liceali, Bontempelli fa riferimento "alla letteratura incosciente e quasi ignorata" presente in ogni secolo, quella nata parallelamente alla letteratura che sarebbe stata poi canonizzata, ma soprattutto quella nata dalle necessità comunicative del quotidiano: memorie, lettere, "[...] scritture pratiche insomma" (Bontempelli, 1913, p. IV). L'arte anche di uso applicativo sarà al cuore del progetto novecentista e Bontempelli la chiamerà "d'uso quotidiano" (Bontempelli 1938, pp. 39-40). Nell'ambito della letteratura una tale produzione è, secondo Bontempelli, "infinitamente più interessante e più vera dell'altra" (Bontempelli, 1913, p. IV). Inoltre, essa nasce in un periodo di "maggior verginità" (Bontempelli, 1913, p. IV). E proprio perché nel frattempo "la letteratura si è chiusa nella rocca illustre del latino e del greco, la volgarità del volgare salva la prosa." (Bontempelli, 1913, p. IV) Quindi, si tratta di una scrittura in un certo senso primitiva proprio perché libera dalle costrizioni e norme linguistiche ed estetiche che valevano nel Quattrocento per la letteratura ufficiale in latino. II divario tra la letteratura ufficiale e quella minore, anche a uso pratico, non sta quindi solo nella lingua (il latino da una parte, il volgare dall'altra), ma anche nel livello di libertà d'espressione (una letteratura cosciente delle proprie modalità e delle proprie ambizioni, da una parte, e una incosciente e spontanea, dall'altra.) Perciò Bontempelli considera il primo Quattrocento "il periodo aureo di tutta la nostra prosa avanti il secolo decimonono [...]" soprattutto perché è un perio do in cui la prosa "servì soltanto alla vita [...]" per i suoi scopi comunicativi, e perciò vi "trovò la spontaneità, il colore e la immediatezza che la fecero arte vera." (Bontempelli, 1913, p. IV) Similmente, nell'articolo "Analogie", prendendo come modello per il rinnovamento del tempo e dello spazio, e di conseguenza della letteratura, la pittura di Mantegna, Piero Della Francesca e Masaccio, Bontempelli nel 1927 scriverà: il novecentismo "[...] 
vive del senso magico scoperto nella vita quotidiana degli vomini e delle cose" (Bontempelli, 1938, p. 36). La pittura dei tre maestri nasce intorno alla quotidianità, al "realismo preciso, avvolto in una atmosfera di stupore lucido" (Bontempelli, 1938, p. 36) che colpisce l'osservatore e lo rende così partecipe del rapporto dialogico con l'opera d'arte.

Uno degli autori che Bontempelli inserisce nella raccolta è Bernardino da Siena, da lui ritenuto "il più grande di tutti i prosatori dei secoli che vanno dal XIII a tutto il XVIII secolo" (Bontempelli, 1913, p. VI). A partire di questa stima per il predicatore nel 1914 gli dedicherà un'intera monografia, dal titolo San Bernardino da Siena, in cui indagherà soprattutto sulla sua arte di narrare spontanea e non-intenzionale, sulla sua abilità a parlare a un pubblico eterogeneo (a volte anche ignorante) di argomenti difficili ma con chiarezza d'espressione, sulla sua fantasia, sulla sua semplicità, sul suo umorismo e soprattutto sulla sua umanità, trovandovi aspetti che avrebbe difeso anche per la letteratura novecentista. II profilo di San Bernardino da Siena è particolare anche perché non presentato cronologicamente e si potrebbe dire che Bontempelli elabora una sorta per niente tradizionale di biografia (si pensi ad esempio a quella di Alfieri). II testo potrebbe, per i suoi contenuti, essere diviso in tre parti. La prima consiste nelle particolarità del carattere di San Bernardino, nel significato che la scrittura significò per lui, nella sfortuna che il predicatore ebbe con i propri compagni di lavoro, i Francescani, a causa di invidie, e nello status di celebrità che egli ottenne nel corso della sua vita. La seconda parte viene dedicata invece alle quarantacinque prediche che San Bernardino svolse nel 1427, che un tale Benedetto di Maestro Bartolomeo riportò interamente su cera. Bontempelli le valuta nel ruolo di critico letterario e da una prospettiva novecentista. L'ultima parte è dedicata alla vita di San Bernardino, però viene raccontata in ordine inverso: Bontempelli inizia a rievocarla dall'ultimo viaggio di San Bernardino per finire con dei fatti sulla nascita

Era nato l'otto di settembre del 1380 (l'anno in cui era morta Santa Caterina); non a Siena propriamente, ma a Massa, che apparteneva al territorio senese; suo padre era Tollo Albizzeschi, chi n'era governatore per la repubblica di Siena (Bontempelli, 1914, p. 69).

Si noti che le considerazioni su alcuni aspetti della scrittura di San Bernardino avanzate nell'Introduzione alle Prose verranno riproposte ed ampliate nella monografia del 1914. Inoltre, il testo del 1914 meriterebbe un esame anche a confronto con gli scritti di Federigo Tozzi e Francesco Flora, che quasi nello stesso periodo si erano interessati al primitivismo misticheggiante di San Bernardino.

Nella raccolta Prose di fede e di vita del primo tempo dell'umanesimo il quaresimale di San Bernardino è riportato quasi interamente soprattutto per quel motivo. Bontempelli considera le prediche "un materiale straordinariamente vivo e vario: aneddoti, favole, ammaestramenti pratici, esortazioni politiche, consigli privati sugli affari di questo o quello degli uditori, barzellette, accenni storici e autobiografici, mille altre cose impreviste" (Bontempelli, 1913, p. V). Curiosamente, come aveva fatto nel 1908 nella recensione alla Canzone dell'olifante di Pascoli e come farà nella monografia su San Bernardino, Bontempelli anche in questa Introduzione sottolinea l'importanza dello schema "su cui ogni predica è costruita" e che in genere è

prettamente scolastico, e secondo la tradizione dei predicatori teologi: una frase di libro sacro n'è il tema; e tutte le parti, le immagini, le parole di quella 
frase, intese nel senso reale e si nel simbolico, danno la partizione su cui la predica si foggia (Bontempelli, 1913, p. V).

Nel 1908 il rimprovero a Pascoli riguardava non solo la mancanza di uno schema, ma anche quello che essa comporta: uno schema permette infatti al lettore di seguire con più facilità il testo poetico ma soprattutto aumenta la possibilità di comprendere ciò che viene detto, e anche di sentirlo (Bontempelli, 1908a, p. 161). Capire un'opera letteraria per Bontempelli non è "un fatto di intelligenza, ma di sensitività. Può entrarvi la intelligenza, ma in quanto aiuti la sensitività, e in essa si risolva" (Bontempelli, 1913, p. V). La sensitività riguarda l'interpretazione individuale di cui parlava già nella sua lectura Dantis nel 1910, inclusa nel volume II canto XXII del Paradiso letto da Massimo Bontempelli nella sala di Orsanmichele, nonché nel testo prefatorio al dramma Fiorenza mia! di Yambo 12, ma soprattutto ne parlerà più tardi, ad esempio nell' articolo "Che cosa è upoesia»" del 1931:

Chi scrive, ha lo scopo di creare nel lettore una serie di sensazioni, di (posso dirlo?) «atmosfere interiori» [...]: I'arte comincia e finisce, non nei pretesti [...] ma negli effetti loro e del loro più o meno armonioso succedersi e variarsi; cioè appunto in quelle «atmosfere interiori», le quali, create appena, vivono per se medesime nell'animo del lettore, e subito gettano via e dimenticano i pretesti (Bontempelli, 1938, pp. 98-99).

Richiamando l'attenzione dei lettori attraverso la domanda retorica "posso dirlo?" e cosciente del fatto che l'espressione atmosfere interiori potrebbe contraddire la sua dichiarata avversione contro l'intimismo e gli psicologismi, Bontempelli mette l'interpretazione individuale in primo piano e la lega fortemente al dovere dello scrittore. Tali effetti possono avere luogo proprio attraverso una precisa arte dello scrivere autonoma e libera dall'adesione a rigidi codici, ma guidata da una struttura.

Nell'incoraggiamento alla lettura, Bontempelli lancia un invito intelligente allo studente liceale: egli si può aspettare da predicatore senese una scrittura basata su motivi tratti dalla quotidianità, raccontati con immaginazione e fantasia; tutto ciò, però, entro una struttura prevedibile sebbene per niente intrusiva e imponente. Bontempelli implicitamente promette al lettore che l'interpretazione dipenderà interamente da colui che legge e non dai suoi maestri. Una libertà nell'approccio ai testi e una democratizzazione della critica si aggiungerà al "calore comunicativo d'una mirabile sincerità di fede amorosa e indulgente [...]" di San Bernardino che avvincerà il giovane lettore e svolgerà allo stesso tempo il compito di favorire la comunicazione (Bontempelli, 1913, p. V). Solo un anno più tardi Bontempelli scriverà sull'effetto che la prosa nel novecentismo dovrebbe avere sui lettori e lo farà tenendo conto anche della prosa di San Bernardino, il quale "[v]uole ancora che, chi può, si scriva qualcosa di ciò che ha udito; che ognuno ne parli poi ad altri, che non fu presente [...] Egli sa benissimo come dev'essere la predica, perché possa essere intesa e fruita così. [...] Bernardino vuole che il suo dire sia inteso: "dirlo chiarozo, chiarozo, acciò che chi ode, ne vada contento e illuminato, e none imbarbagliatoı" (Bontempelli, 1914, pp. 55-56). Per dirla con le parole di "un cronista [...] contemporaneo", ascoltando le prediche di San Bernardino: "uci pareva d'essere tutti santi, avendo buona devoziones" (passo citato in Bontempelli, 1913, p. V).

In linea con le sue posizioni anticanoniche e in difesa di fasi letterarie di nuovo inizio, Bontempelli esclude dalla raccolta "le cronache propriamente dette, in quanto il

\footnotetext{
12 Anche la prefazione al dramma Fiorenza mia! di Yambo (Enrico Novelli) entra nel corpus di testi in analisi nella ricerca dottorale in corso.
} 
cronista è già un po' costruttore, raccoglie e ordina i fatti con qualche intenzione studiosa se non letteraria" (Bontempelli, 1913, p. VI). Per la sezione appunto delle cronache sceglie invece quelle nate da esigenze pratiche. Sono i casi di Buonaccorso Pitti e di Giovanni Morelli, "due tipi diversissimi", e sono tali anche le loro prose: quella di Buonaccorso Pitti risulta "disadorna e frizzante", mentre quella di Morelli è "un po' zoppicante ne' costrutti, commovente per la semplice sincerità degli affetti" (Bontempelli, 1913, pp. VI-VII). L'unica donna rappresentata in questa raccolta è Alessandra Macinghi-Strozzi con le sue lettere "ai figli esuli da Firenze: pensieri e stile alti, semplici, mestamente sereni" (Bontempelli, 1913, p. VII). Più attenzione viene dedicata alle lettere "ai dieci di Balia" di Rinaldo degli Albizi, valutando soprattutto lo stile "schiettamente fiorentino, arguto, disinvolto, incisivo", tratti che saranno decisamente propri del novecentismo e su cui Bontempelli insisterà persino negli anni Trenta. ${ }^{13}$

La conclusione dell'Introduzione alle Prose sembra presa da una lezione liceale per le sue caratteristiche retoriche, come ad esempio la ripetizione di certe nozioni menzionate nel testo'14, l'inversione dell'ordine delle parole per dare l'enfasi al discorso ${ }^{15}$ e dove si osserva pure l'attitudine a cominciare le frasi con un pronome relativo ${ }^{16}$ o con una congiunzione, come e o $\mathrm{ma}^{17}$, fenomeno che ricorda non solo la lingua parlata, ma anche un certo stile giornalistico. Lo stesso vale per le frasi impersonali, ad esempio: "E quanto al tempo si attiene alla prima metà del quattrocento. [...] II limite è, come tutti i limiti di questo genere, artificioso, ma era pur necessario metterne uno" (Bontempelli, 1913, p. VI.) Inoltre, Bontempelli dimostra di essere consapevole del pubblico per cui scrive: giovani liceali che potrebbero essere restii a studiare le opere imposte dal curriculum e restare invece affascinati da autori minori la cui opera si connota appunto per maggiore spontaneità. Anche il tono della prefazione merita attenzione: lascia passare infatti un giudizio favorevole verso le opere degli autori proposti (soprattutto verso Bernardino da Siena), giudizio avvalorato anche dal fatto che non si trattava di opere rientranti nel canone letterario del primo Quattrocento. Alla fine della Introduzione è inserita poi una lista aggiuntiva di indicazioni per chi fosse stato interessato ad approfondimenti (vi si trovano sia testi critici di altri autori, sia opere ulteriori degli scrittori inclusi nella raccolta). La presentazione bontempelliana, nonostante giunga a un bilancio sui punti chiave, risulta fornita di una conclusione aperta, proprio per quella lista di indicazioni supplementari inseritevi alla fine che fungono da invito alla lettura di altre opere e ad approfondimenti. In quel lavoro si sostiene l'importanza di un confronto con quella letteratura "incosciente e ignorata"18, rappresentata da opere che sono uno

\footnotetext{
13 In un articolo intitolato "Inaugurazione del «Teatro Reale dell'Opera» ovvero i funerali del melodramma", secondo Bontempelli la letteratura della Terza epoca dovrà essere guidata dalla metafora della "illuminazione sfarzosa e la luce chiara" (Bontempelli, 1938, pp. 357-358).

14 "La raccolta esclude, come ho detto e per la ragione che ho detto, tutti gli autori d'intenzioni letterarie [...]" (Bontempelli, 1913, p. VII).

15 "Di una larga scelta dal Quaresimale del 1427 è fatta la maggior parte di questo libro." (Bontempelli, 1913, p. V.) e "Della vita del beato Colombini scriveva il Giordani [...]" (Bontempelli, 1913, p. VI.).

16 "La quale trova la sua più candida espressione nelle lettere che Alessandra Macinghi-Strozzi [...] scrisse [...]" (Bontempelli, 1913, p. VII).

17 "Ed ecco in fine un' altra specie di scritture pratiche [...]" (Bontempelli, 1913, p. VII). E ancora: "Ma nelle cronache della vita pubblica erano spesso commisti memoriali privati [...]" (Bontempelli, 1913, p. VI).

18 "E quanto al tempo si attiene alla prima metà del [Q] uattrocento. [...] II limite è, come tutti i limiti di questo genere, artificioso, ma era pur necessario metterne uno" (Bontempelli, 1913, p. IV).
} 
specchio chiaro e trasparente dello spirito dell'epoca e che, proprio perché ancora non canonizzate, meglio si prestano a un'interpretazione soggettiva da parte dei lettori.

\section{Conclusioni}

Dalla analisi fin qui condotta traspare che alcune posizioni del novecentismo si possono trovare all'interno della rivista «900» in testi pubblicati oltre dieci anni prima dell'ufficiale lancio di tale tendenza. Pensiamo a posizioni come quella che evita demarcazioni rigide tra la prosa e poesia o ancora a quando, nella considerazione della qualità dei testi, Bontempelli supera le distinzioni fra opere canoniche e opere non canoniche. L'autore dimostra, ad esempio, la validità di opere in volgare del primo Quattrocento spesso ingiustamente trascurate. Secondo lui queste sarebbero l'espressione più perfetta dello spirito del tempo di quel secolo. Al proposito, da questo ragionamento deriva anche la predilezione di Bontempelli per autori minori. Si potrebbe dire che più le opere sono considerate minori, più grande è secondo lui la loro espressività. E ciò emerge chiaramente sia nella Introduzione alle Prose di fede e di vita del primo tempo dell'umanesimo che negli articoli dei primi anni Venti e degli anni Trenta qui esaminati. Questa letteratura incosciente è slegata dalle regole del canone e perciò nasce su un terreno parallelo, spesso costituito da scritture private. Nel valore di tali scritti avrebbe un ruolo importante anche l'ispirazione che li guida e che si oppone a un tipo di attività letteraria condotta per motivi pratico-economici. Inoltre, dai testi esaminati emerge la predilezione per uno stile chiaro, trasparente e svincolato da ogni estetismo: proprio lo stile delle lettere della Macinghi-Strozzi oppure dei saggi sulla vita di Rinaldo Degli Albizi, servirebbero da modello per la scrittura novecentista, la quale si ispirerà per quelle stesse ragioni alla scrittura praticata nel campo del giornalismo. Bernardino da Siena viene presentato non solo come il predicatore par excellence, ma anche come un maestro del dialogo, capace di impreviste interazioni con i suoi lettori, verso i quali si rapporta con attitudine rispettosa o quasi affettuosa e attraverso un linguaggio chiaro e lucido, comprensibile a tutti. Attraverso quattro autori contemporanei, Bontempelli, nell'articolo del 1922, dimostra invece quanto la letteratura italiana sia, all'epoca, ancora lontana da qualsiasi rinnovamento e auspica implicitamente che lo spazio letterario italiano adotti un approccio capaci portare a uno svecchiamento. Come nota Ugo Piscopo, negli anni Venti Bontempelli fisserà, "in nome della massa [...] [i] punti essenziali della sua poetica" fra cui

la trasparenza linguistica [...], la dilatazione del teatro a spettacolo così uda far delirare un miliardo di persone», la polemica contro l'intellettualismo sofisticato e la disponibilità ad accogliere, invece, «la stupidità del pubblico, che di tanto in tanto salva la purità e la sanità e la verginità dell'arteı (Piscopo, 2001, pp. 391-392).

\section{Bibliografia}

ARIOSTO, L. (1916). Commedie e satire - con una prefazione di Massimo Bontempelli. Milano: Istituto Editoriale Italiano, pp. 9-26.

BARETTI, G. (1914). La frusta letteraria - con una prefazione di Massimo Bontempelli. Milano: Istituto Editoriale Italiano, pp. 9-23. 
BLACK, R. (1992). Florence. In BLACK, R. \& PORTER, R. (ed.), The Renaissance in National Context. Cambridge: Cambridge University Press.

BONTEMPELLI, M. (1908a). Cronache di poesia. Rassegna contemporanea, a. 1, fasc. 7, pp. 159-163.

BONTEMPELLI, M. (1908b). Grande e piccola critica. Rassegna contemporanea, a. 1, fasc. 2, pp. 128-140.

BONTEMPELLI, M. (1910a). Il canto XXII del Paradiso letto da Massimo Bontempelli nella sala di Orsanmichele. Firenze: Sansoni.

BontempelLI, M. (1910b). II Poliziano. II Magnifico. Lirici del Quattrocento - scelta e commento di Massimo Bontempelli. Firenze: Sansoni.

BONTEMPELLI, M. (1910c). (8 maggio 1910). Un nuovo e un antico. II Marzocco, 19, a. XV, pp. 2-3.

BONTEMPELLI, M. (1910d). (29 maggio 1910). Fuori della letteratura. II Marzocco, 22, a. $X V$, p. 4.

BONteMPELl, M. (1910e). (26 giugno 1910). In difesa dei pedanti: Per un libro di F. D'Ovidio. II Marzocco, 26, a. XV, pp. 3-4.

BONTEMPELLI, M. (ed.). (1913). Prose di fede e di vita del primo tempo dell'umanesimo. Scelta e commento di Massimo Bontempelli. Firenze: Sansoni.

BONTEMPELLI, M. (1914). San Bernardino da Siena. Genova: A. F. Formíggini.

BONTEMPELLI, M. (il 7 luglio 1922). Cronache della prosa - Cercando la fantasia. II Mondo.

BONTEMPELLI, M. (1938). L'Avventura novecentista, Selva polemica (1926-1938). Firenze: Vallecchi.

BONTEMPELLI, M. (2009). La vita intensa. Milano: ISBN Edizioni.

Croce, B. (1905). Varietà. La Critica. Rivista di Letteratura, Storia e Filosofia diretta da B. Croce. No. 3, 1905.

CROCE, B. (1908). Estetica come scienza dell'espressione linguistica generale: teoria e storia. Bari: Laterza.

GELLI, G. B. (1915). Scritti. Milano: Istituto Editoriale Italiano.

GenetTE, G. (1989). Soglie. I dintorni del testo. Torino: Einaudi.

PISCOPO, U. (2001). Massimo Bontempelli. Per una modernità dalle pareti lisce. Liguori: Napoli.

RAGONE, G. (1983). Dal libro manoscritto all'editoria di massa. In AsOR RosA, A. (ed.), Letteratura italiana, 2 vol. Produzione e consumo. Torino: Einaudi.

StendHAl (1913). Il rosso e il nero. Traduzione e introduzione di Massimo Bontempelli. Milano: Istituto Editoriale Italiano.

TRANFAGLIA, N. (2000). L'editoria nell'Italia contemporanea: sviluppo e peculiarità negli ultimi cento anni. In TRANFAgLIA, N. \& VITTORIA, A., Storia degli editori italiani. Bari: Laterza. 